\title{
Registro de evaluación de tratamiento de cáncer gástrico en Chile (REGATE): Características clínicas basales de 523 pacientes*
}

\author{
Drs. BETTINA MÜLLER ${ }^{1}$, HERNÁN DE LA FUENTE H. ${ }^{2}$, OLGA BARAJAS B. ${ }^{3}$, \\ BERNARDITA CARDEMIL J. ${ }^{4}$, ANTONIO VILA T. ${ }^{5}$, EDUARDO MORDOJOVICH S. ${ }^{6}$, \\ KARINA PEÑA N. ${ }^{7}$ CÉSAR CASTILLO C. ${ }^{8}$, MÓNICA CAMPOS M. ${ }^{9}$, \\ HERNÁN ROJAS R. ${ }^{10}$, INGRID QUIJADA P. ${ }^{11}$, CRISTIÁN YÁÑEZ R. ${ }^{12}$ \\ 1 Oncóloga Médica. Instituto Nacional del Cáncer, Santiago. \\ 2 Fundación Arturo López Pérez, Santiago. \\ 3 Hospital Clínico Universidad de Chile, Santiago. \\ ${ }^{4}$ Hospital Base de Valdivia, Valdivia. \\ 5 Hospital Regional de Concepción, Concepción. \\ 6 Hospital Regional de Puerto Montt, Puerto Montt. \\ 7 Hospital San Juan de Dios, Santiago. \\ 8 Hospital Barros Luco, Santiago. \\ 9 Hospital San Borja Arriarán, Santiago. \\ ${ }^{10}$ Hospital Sótero del Río, Santiago. \\ ${ }^{11}$ Instituto Clínico Oncológico del Sur ICOS, Temuco. \\ ${ }^{12}$ Sanofi-Aventis de Chile. \\ Chile
}

\begin{abstract}
Registry of gastric cancer evaluation in Chile (REGATE): Basal clinical features of 523 patients

Background: Gastric cancer is one of the leading causes of cancer-related deaths in Chile and worldwide. No consensus exists for therapeutic management. Aim: To assess clinical features and practice patterns of patients with newly diagnosed gastric cancer in Chile. Method: Chilean patients $>18$ years old with newly diagnosed primary gastric adenocarcinoma enrolled by thirteen centers from different regions of Chile. Target sample size calculated according to gastric cancer prevalence in Chile. Data collected from two visits within a 10-month timeframe: baseline (patients and tumor features, treatment plan) and end of study (completion of initial treatment). Herein, baseline visit data is presented. Results: Between 2005 and 2008, 523 patients enrolled. Median age 61.3 years. Diagnosis by endoscopy in $98.5 \%$ patients. Location: body $35.8 \%$, proximal $35.4 \%$, and antral $23.9 \%$. Most frequently used histopathological classification was WHO classification, with tubular adenocarcinoma being most frequent finding (53.1\%). AJCC/UICC clinical staging (available in 37.7\% of patients) was: 0 and I $-23.3 \%$, II - $18.3 \%$, III- $20.8 \%$, IV - 37.6\%. Therapeutic choice based mainly on
\end{abstract}

*Recibido el 8 de Mayo de 2010 y aceptado para publicación el 14 de Septiembre de 2010.

Fuente de apoyo financiero: Estudio patrocinado por Sanofi-Aventis intercontinental, quien no tuvo influencia en el análisis de los resultados.

Correspondencia: Dra. Bettina Müller.

Profesor Zañartu 1010, Santiago, Chile.

bgmuller@gmail.com 
clinical staging (49.9\%) and included surgery in 440 patients $(84.1 \%)$. Therapy planned by surgeon $(54.9 \%)$ or multidisciplinary team $(42.3 \%)$. Conclusions: REGATE is the largest prospective multicenter registry study performed in Chile. Basal visit data report that diagnosis is established frequently at advanced stages. Surgery is the most frequent therapeutic choice, (neo-) adjuvant therapies are only planned in one out of four patients. End of study visit data will provide the full scope of diagnosis and treatment of these patients.

Key words: Stomach neoplasms, neoplasms staging.

\section{Resumen}

Introducción: El cáncer gástrico es una de las principales causas de muerte por cáncer en Chile. No existe consenso acerca del tratamiento. Objetivos: Conocer características clínicas y patrón de tratamiento de pacientes con cáncer gástrico recién diagnosticado. Material y Método: Pacientes chilenos mayores de 18 años con diagnóstico reciente de adenocarcinoma gástrico primario, enrolados en 13 centros de diferentes regiones de Chile. Datos obtenidos en dos visitas dentro de período de 10 meses: basal (características del tumor y paciente, plan de tratamiento) y fin de estudio (tratamiento inicial completado). Se presentan datos de visita basal. Resultados: Entre 2005 y 2008, 523 pacientes enrolados. Mediana edad 61,3 años. Diagnóstico por endoscopia en 98,5\% pacientes. Localización: corporal 35,8\%, proximal 35,4\% y antral 23,9\%. Clasificación histopatológica más usada fue clasificación OMS, y tipo histopatológico más frecuente fue tubular 53,1\%. Etapificación clínica AJCC/UICC (disponible en 37,6\% de pacientes) distribuida en: 0 y I - 23,3\%, II - 18,3\%, III - 20,8\%, IV - 37,6\%. Principal característica clínica para elección de terapia planeada fue etapificación clínica $(49,9 \%)$. Plan de tratamiento consideró cirugía en 440 pacientes $(84,1 \%)$. En mayoría de casos, plan terapéutico decidido por cirujano $(54,9 \%)$ o equipo multidisciplinario $(42,3 \%)$. Conclusiones: REGATE es el estudio de registro prospectivo multicéntrico más grande desarrollado en Chile. Datos visita basal informan que diagnóstico se establece frecuentemente en etapas avanzadas. Cirugía es alternativa terapéutica más frecuentemente indicada; terapias (neo-) adyuvantes sólo son ofrecidas a uno de cuatro pacientes. Datos visita fin de estudio proveerá visión completa del diagnóstico y tratamiento de estos pacientes.

Palabras clave: Neoplasias gástricas, etapificación de neoplasias.

\section{Introducción}

El cáncer gástrico en el mundo constituye la segunda causa de muerte por cáncer ${ }^{1}$. Chile es uno de los países con más alta incidencia mundial de este tipo de cáncer, junto con Japón, Costa Rica y Singapur $^{2}$. En Chile, es la primera causa de muerte por cáncer en hombres (tasa de mortalidad de 24,9 por 100.000); en mujeres, es la tercera causa de muerte por cáncer después del cáncer de vesícula biliar y de mama (tasa de mortalidad de 12,2 por 100.000$)^{3}$. $\mathrm{Su}$ tasa de mortalidad cruda se ha mantenido estable desde la década de los 80 , fluctuando entre 19 a 20 por 100.000 habitantes, determinando alrededor de 3.000 muertes por año ${ }^{4}$.

Uno de los factores de riesgo más estudiados en los últimos años es la infección por Helicobacter pylori, que se ha podido establecer como factor carcinogénico para el cáncer gástrico ${ }^{5}$.

El cáncer gástrico se diagnostica en etapas avanzadas en la mayoría de los países, con excepción de Japón, donde el cáncer gástrico incipiente (etapa I) se diagnostica en más del $50 \%$ de los sujetos, con una supervivencia a 5 años cercana al $90 \%{ }^{6}$.

La resección quirúrgica con criterio oncológico es parte esencial del tratamiento curativo del cáncer gástrico localmente avanzado. La disección D2 se ha convertido en la cirugía estándar para cánceres localmente avanzados ${ }^{7}$. En el cáncer gástrico avanzado resecado (Etapa IB-IVA), la supervivencia a 5 años no supera el $50 \%$ por lo que se han explorado terapias adyuvantes y neoadyuvantes con Quimioterapia y/o Radioterapia. Las estrategias de Quimioterapia perioperatoria, Quimioradioterapia postoperatoria y Quimioterapia adyuvante, han podido establecer un beneficio significativo en la supervivencia a 5 años en estudios clínicos randomizados fase III $^{8-10}$ y se consideran terapias estándar hoy en día ${ }^{11}$. En el cáncer gástrico metastásico o irresecable, se ha demostrado que la Quimioterapia paliativa aumenta la supervivencia mediana y mejora la calidad de vida ${ }^{12}$.

No obstante lo anterior, la introducción de estos avances a la práctica clínica habitual, se ha visto entorpecida porque no existe un consenso con relación al tratamiento estándar y la estrategia utilizada varía de una región a otra. Así, por ejemplo, en Estados Unidos se adoptó ampliamente la Quimioradioterapia postoperatoria, mientras que en Europa existe un consenso acerca del uso de la Quimioterapia perioperatoria. En los países asiáticos en cambio, la Quimioterapia adyuvante está siendo utilizada ampliamente hoy en día. En Chile, muchos pacientes son tratados sólo con cirugía, mientras que en otros se ha utilizada la Quimioradioterapia postoperatoria. 
El Estudio REGATE (Registro de Evaluación de Tratamiento del Cáncer Gástrico) es un estudio observacional internacional que fue desarrollado bajo la conducción de un consejo asesor científico compuesto por seis miembros académicos con experiencia en investigación y práctica clínica en cáncer gástrico. Se realizó en 250 centros en 27 países, reclutando más de 10.000 pacientes con cáncer gástrico a nivel mundial entre agosto de 2004 y julio de 2008. Este estudio fue diseñado para describir el patrón del manejo médico de pacientes con cáncer gástrico recién diagnosticados en cualquier etapa de la enfermedad. En este artículo se presenta el análisis de las características clínicas basales de un grupo de pacientes chilenos incorporados a este registro.

\section{Material y Método}

Este estudio fue conducido de acuerdo a los estándares aceptados globalmente de Buenas Prácticas Clínicas (según lo definido en ICH E6 Guidelines for Good Clinical Practice (GCP), mayo 1996), de acuerdo a la Declaración de Helsinki y las regulaciones locales. El protocolo y el formulario de información para el paciente y consentimiento informado fueron aprobados por el Comité de Ética ad-hoc del Ministerio de Salud de Chile en noviembre de 2005, de acuerdo al marco regulatorio legal local.

Para la selección de la muestra, se consideró un método de muestreo de dos niveles: centros y pacientes. El tamaño de muestra local fue calculado de acuerdo a la prevalencia estimada de cáncer gástrico en Chile, en base a lo cual se planeó enrolar 454 pacientes chilenos en el estudio REGATE. Del total de centros que tratan pacientes con cáncer gástrico en Chile, se seleccionaron en forma aleatoria trece sitios en seis ciudades de Chile (Antofagasta, Santiago, Concepción, Temuco, Valdivia y Puerto Montt), incluyendo 9 establecimientos del sector público y 4 establecimientos del sector privado. La distribución por especialidad de los investigadores locales fue: 2 cirujanos, 2 oncólogos radioterapeutas y 9 oncólogos médicos. Los pacientes consecutivos con un diagnóstico nuevo de cáncer gástrico fueron considerados elegibles para enrolamiento por el investigador local del estudio. Todos los pacientes debían cumplir con los criterios de inclusión/exclusión para poder ser enrolados. Los criterios de inclusión se establecieron como: varones o mujeres ${ }^{3}$ de 18 años, adenocarcinoma gástrico primario, paciente recientemente diagnosticado, haber otorgado su consentimiento informado por escrito. Los criterios de exclusión fueron: antecedentes de otra neoplasia, excepto el cáncer de piel de tipo no melanoma tratado curativamente o carcinoma del cuello del útero in-situ tratado adecuadamente.
La información fue recolectada en Formularios de Reporte de Casos (CRFs) en dos visitas dentro de un período de 10 meses: visita de inclusión y visita de fin de estudio. En la visita de inclusión se recolectaron las características del paciente y del tumor al momento del diagnóstico y el tratamiento planificado; y en la visita de fin de estudio, se evaluaron estas características al término del tratamiento inicial planificado. En Chile, se enrolaron pacientes desde diciembre de 2005 a septiembre de 2008 . El presente artículo describe las características de los pacientes chilenos en la visita de inclusión.

\section{Resultados}

Entre diciembre de 2005 y septiembre de 2008, un total de 545 pacientes fueron enrolados al estudio REGATE por trece centros clínicos. Del análisis se excluyeron 22 pacientes, siete por no presentar cáncer, 7 por no cumplir con todos los criterios de inclusión, 6 por presentar otras neoplasias, y dos por no estar disponible la ficha clínica. En total se analizaron 523 pacientes, con una mediana de 37 pacientes por centro. Las características de estos pacientes se resumen en la Tabla 1.

De estos pacientes, $8(1,5 \%)$ se encontraba participando en otro estudio clínico al momento de ser enrolado en este estudio.

La distribución etaria de los pacientes se ilustra en la Figura 1, revelando que cerca del $20 \%$ de los pacientes tenían menos de 50 años.

Los síntomas gástricos estaban presentes en 478 pacientes $(91,4 \%)$ y la mediana de duración de síntomas hasta el diagnóstico fue de 3 meses, con un rango entre $<1$ y 312 meses.

El diagnóstico se realizó por endoscopia en la gran mayoría de los pacientes $(98,5 \%)$, pero la presencia o ausencia de Helicobacter pylori se conocía sólo en el 30\% de los pacientes. Entre éstos, el 40\% era portador de esta bacteria (64/157).

Las principales localizaciones del tumor primario eran corporal $(35,8 \%)$ y proximal $(35,4 \%)$, seguido por las lesiones antrales $(23,9 \%)$ y del compromiso extenso gástrico en 26 pacientes $(5 \%)$.

El tipo histopatológico se encontraba disponible en 461 pacientes $(88,1 \%)$ en la visita de inclusión. El tipo de clasificación más usado fue la clasificación de la OMS, que se usó en forma exclusiva o combinada en $84 \%$ de los casos. Otras clasificaciones usadas fueron la de Lauren (37\%), o de Ming $(25 \%)$. El tipo histológico más frecuente fue el adenocarcinoma tubular (52\%), seguido por el adenocarcinoma de células en anillo de sello (27\%). Según la clasificación de Lauren, el tipo histológico más frecuente fue el adenocarcinoma de tipo intestinal 
B. MÜLLER y cols.

Tabla 1. Características de pacientes enrolados en REGATE

\begin{tabular}{ll}
\hline Características de los pacientes & $\mathbf{n}=\mathbf{5 2 3}$ \\
\hline Edad promedio (rango) & 61,3 años (27-93) \\
Proporción de hombres, n (\%) & $67,5 \%$ \\
Presencia de síntomas gástricos & $91,4 \%$ \\
Tiempo desde primeros síntomas & 8,1 meses (promedio) \\
& 3,0 meses (mediana) \\
& Rango $0,03-312,0$ meses \\
Historia familiar de cáncer gástrico & $7,8 \%$ \\
Infección confirmada por Helicobacter pylori & $12,2 \%$ \\
\hline
\end{tabular}

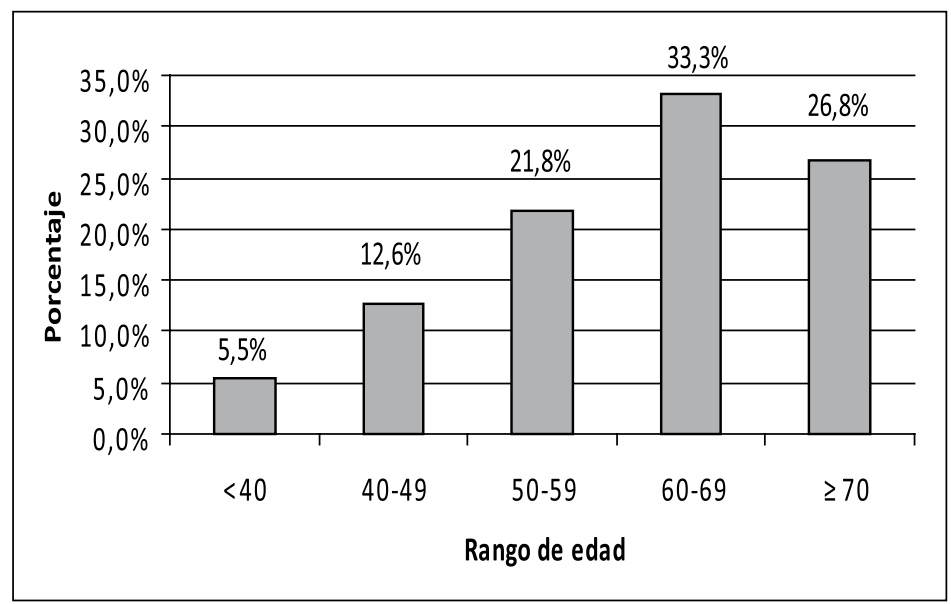

Figura 1. Distribución etaria al diagnóstico.
$(36 \%)$, seguido por el tipo difuso (32\%) (Tabla 2).

El grado histopatológico se encontraba disponible en 403 pacientes en la visita de inclusión. Cerca de la mitad de estos pacientes $(49,4 \%)$ tenía tumores pobremente diferenciados o indiferenciados (Figura 2).

Se reportó la etapificación clínica en la visita de inclusión sólo en 202 pacientes $(38,6 \%)$. El sistema de etapificación más usado fue el de la $\mathrm{AJCC} / \mathrm{UICC}(97,6 \%)$, mientras que la clasificación japonesa se usó en 72 pacientes $(35,7 \%)$. Considerando la etapificación según $\mathrm{AJCC} /$ UICC, más de un tercio de los pacientes $(37,6 \%)$ fueron diagnosticados en cáncer gástrico en etapa IV (Figura 3).

Los métodos de evaluación más frecuentemente utilizados para la etapificación clínica fueron la Tomografía Axial Computada (TC) $(83,5 \%)$, el examen físico $(77,6 \%)$ y la ecotomografía abdominal $(33,2 \%)$. Los factores que más influyeron en la decisión terapéutica fueron la etapificación (50\%), la localización del tumor $(32,2 \%)$, y en menor medida, la edad $(9,4 \%)$ y el estado general del paciente $(4,6 \%)$.

El plan de tratamiento estaba disponible en el total de la muestra

Tabla 2. Histopatología de lesiones

\begin{tabular}{|c|c|c|c|c|c|}
\hline \multicolumn{6}{|c|}{$\begin{array}{c}\text { Disponibilidad de tipo histopatológico } \\
n=461(88,1 \%) \\
\text { (pacientes pueden ser incluidos en más de una clasificación) }\end{array}$} \\
\hline \multicolumn{2}{|c|}{$\begin{array}{c}\text { Clasificación OMS } \\
\mathbf{n}=\mathbf{3 8 7}(\mathbf{8 4 \%})\end{array}$} & \multicolumn{2}{|c|}{$\begin{array}{l}\text { Clasificación de Lauren } \\
\qquad \mathbf{n}=171(37 \%)\end{array}$} & \multicolumn{2}{|c|}{$\begin{array}{l}\text { Clasificación de Ming } \\
n=116(25,2 \%)\end{array}$} \\
\hline Tubular & $68,1 \%$ & Intestinal & $49,1 \%$ & Infiltrante & $100 \%$ \\
\hline Anillo Sello & $27,7 \%$ & Difuso & $44,4 \%$ & Expansivo & $0 \%$ \\
\hline Otro & $11,8 \%$ & Mixto & $6,5 \%$ & & \\
\hline Papilar & $3,4 \%$ & & & & \\
\hline Indiferenciado & $2,4 \%$ & & & & \\
\hline
\end{tabular}




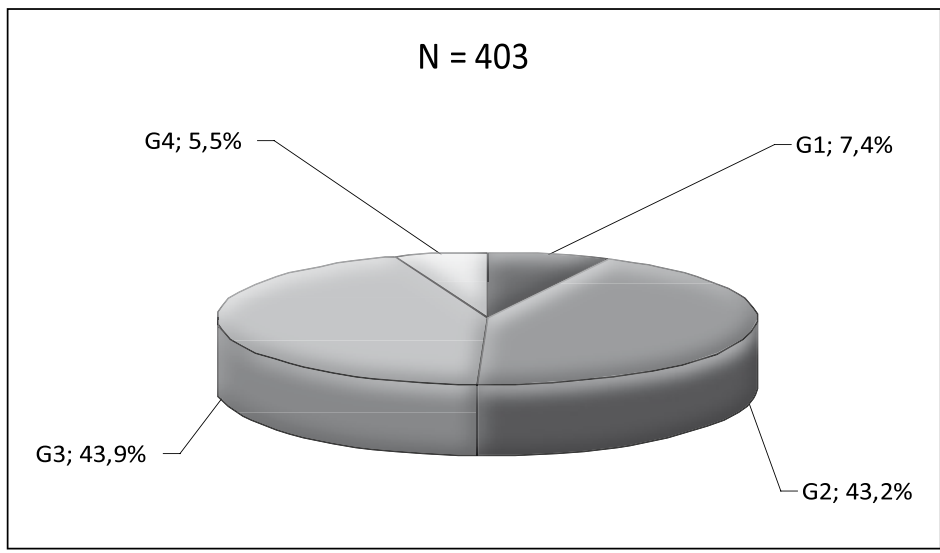

Figura 2. Grado histopatológico.

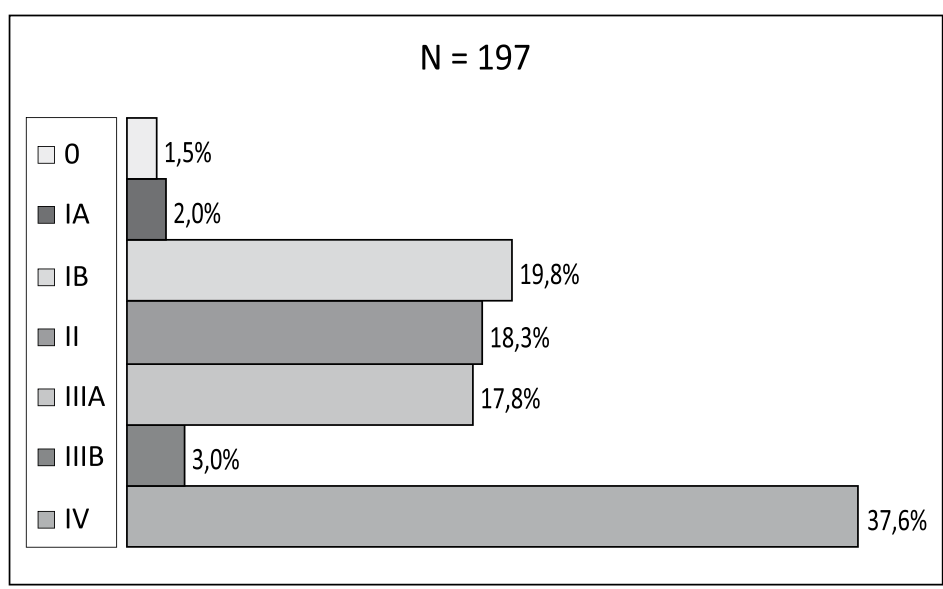

Figura 3. Estadio clínico (AJCC/UICC).

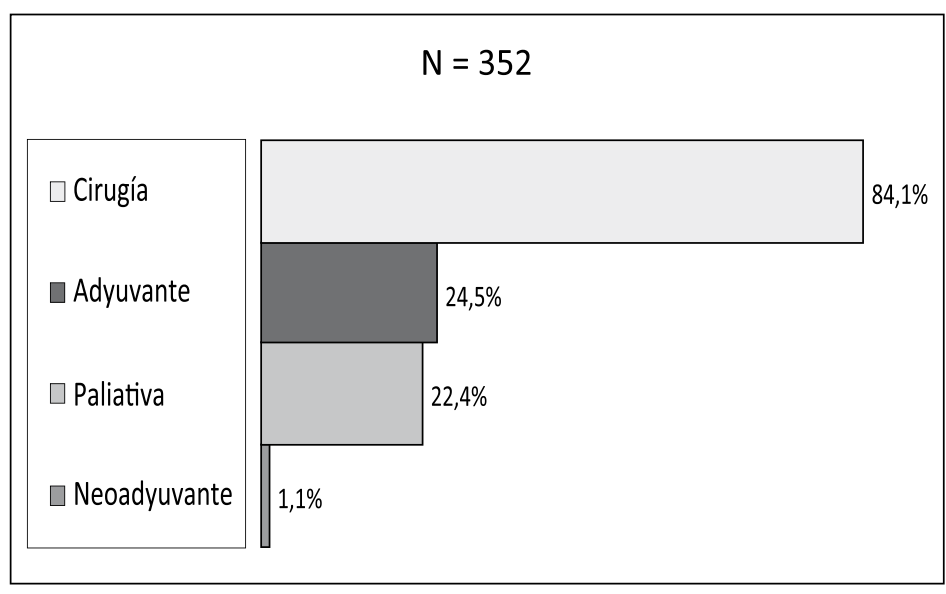

Figura 4. Plan de tratamiento. e incluía la cirugía en el $84,1 \%$, la terapia adyuvante y neoadyuvante estaba planeada en un 24,5 y un $1,1 \%$, respectivamente (Figura 4).

Consultados respecto a las especialidades médicas involucradas en la decisión del plan de tratamiento, éste se basó en la decisión del cirujano en un $54,9 \%$ y en la recomendación de un Comité Multidisciplinario en un $42,3 \%$.

\section{Discusión}

El estudio REGATE es el registro prospectivo multicéntrico internacional más grande realizado en cáncer gástrico. La participación de Chile fue muy exitosa, ya que se llegó al número propuesto de pacientes enrolados, representando el $6^{\circ}$ país con más pacientes incluidos al estudio. Este primer análisis, que reporta los datos de la visita de inclusión, permite evaluar las características demográficas y clínicas de los pacientes al momento del diagnóstico y las consideraciones asociadas a la elección del plan de tratamiento. La edad mediana de 61,3 años es muy similar a la reportada en el análisis global de REGATE que reportó una edad mediana de 60 años ${ }^{13} \mathrm{y}$ algo menor al registro de Valdivia publicado por Heise que observó una edad mediana de 66,8 años ${ }^{14}$.

Concordante con la serie mundial, que reportó la presencia de síntomas gástricos en el $90 \%$ de la población estudiada, la gran mayoría de nuestros pacientes estaban sintomáticos al momento del diagnóstico. La duración de síntomas previo al diagnóstico, es consistente con los hallazgos del registro de Valdivia, en el cual el 40\% de los pacientes habían presentado síntomas por más de 6 meses previo al diagnóstico y podría reflejar la falta de conciencia de la población acerca de la necesidad de consultar al médico en caso de síntomas digestivos y la baja accesibilidad a atención médica especializada.

El principal método de diagnós- 
tico fue la endoscopia digestiva alta, utilizada en el $98,5 \%$ de los pacientes. En ese contexto llama la atención la falta de determinación del Helicobacter pylori, desconocido en el $70 \%$ de nuestra serie.

Las cifras sobre la localización del tumor revelan un aumento de las localizaciones proximales con una disminución de las lesiones antrales en comparación con series chilenas publicadas en los años 70 , lo cual revela una tendencia similar a la observada en los países de América del Norte y de Europa ${ }^{15}$. En comparación con la serie mundial se observa un porcentaje menor de lesiones antrales ( 24 y $39 \%$ ).

El tipo histopatológico se encontraba estudiado en un porcentaje importante de pacientes $(88,1 \%)$, lo cual es concordante con el diagnóstico endoscópico y la confirmación histológica en la gran mayoría de los pacientes. La clasificación histológica más usada fue la clasificación de la OMS, pero también hubo informes anatomopatológicos basados en la clasificación Lauren, o una mezcla de ambos, lo cual dificulta el análisis de la serie.

Un alto porcentaje $(37,6 \%)$ de los pacientes en la serie chilena fue diagnosticado en una etapa clínica avanzada, un hallazgo que se repite en el estudio REGATE global que reportó el 37,7\% de los pacientes diagnosticados en estadio IV. Lo mismo se puede observar en la serie publicada por Heise ${ }^{14}$ que muestra cifras incluso mayores $(56,1 \%)$, y en los datos publicados por García y colaboradores, quienes mostraron que en una serie de 423 pacientes tratados quirúrgicamente, $45,9 \%$ correspondió a un estadio IV $^{16}$.

El método clínico más frecuentemente empleado para la etapificación fue la TAC $(83,5 \%)$, un porcentaje incluso mayor que la serie REGATE global, que reportó el uso de TAC en el 75,7\%, lo cual podría reflejar un cierto grado de selección de los pacientes por haber sido enrolados en centros de atención terciaria, ya que se contrapone a los hallazgos descritos en el registro poblacional de Valdivia, en el cual sólo un $21,6 \%$ de los pacientes habían sido estudiados con TC.

Los factores más frecuentemente considerados para la decisión terapéutica fueron la etapificación clínica y la localización del tumor, similar a lo descrito en la serie global.

Interesantemente, la edad del paciente no incidió importantemente en la decisión terapéutica.

El plan de tratamiento fue decidido por un cirujano en más de la mitad de los pacientes, a pesar de que los investigadores eran mayoritariamente oncólogos médicos, pero es destacable que en un porcentaje importante $(42,3 \%)$, esta fue basada en la evaluación por un equipo multidisciplinario que decidió la modalidad y secuencia terapéutica, cifra muy similar a lo que ocurre en el resto del mundo.
En conclusión, REGATE es el estudio de registro prospectivo multicéntrico de mayor tamaño muestral desarrollado en Chile. Los datos obtenidos permiten afirmar que el cáncer gástrico sigue siendo diagnosticado luego de un tiempo prolongado con síntomas presentes y en etapas avanzadas. El diagnóstico se realiza por endoscopia con confirmación histológica en la gran mayoría de los casos. Este estudio permitió confirmar que la cirugía forma parte del plan de tratamiento en la gran mayoría de los casos, mientras que las terapias adyuvantes o neoadyuvantes sólo son ofrecidas a 1 de cada 4 pacientes diagnosticados, no obstante, cerca de la mitad de los pacientes fueron evaluados por un Comité Multidisciplinario para establecer el plan terapéutico.

Los datos de la visita de fin de estudio permitirán completar el cuadro general de lo que ocurre con el cáncer gástrico y su tratamiento en Chile.

\section{Referencias}

1. Crew K, Neugut A. Epidemiology of gastric cancer. World J Gastroenterol. 2006; 12:354-62.

2. Parkin D. Global Cancer Statistics 2002 CA Cancer J Clin. 2005;55:74-108.

3. Departamento de Estadísticas e Información de Salud, Ministerio de Salud de Chile: Mortalidad por tumores malignos del estómago según sexo, 1990-2007. Versión online consultada 02 de enero de 2009 en http://163.247.51.54/nuev/salida/estomago_1990_2007. php

4. Calvo A. Pesquisa poblacional de cáncer gástrico en pacientes sintomáticos digestivos, período 1996-2000. Rev Med Chile 2001;129. Versión online consultada 07 de octubre de 2009. en http://www.scielo.cl/scielo.php? pid=S003498872001000700007\&script=sci_arttex

5. Helicobacter and Cancer Collaborative Group: Gastric cancer and Helicobacter pylori: a combined analysis of 12 case control studies nested within prospective cohorts. Gut 2001;49:347-53.

6. Nio Y. Comparison of Survival Curves of Gastric Cancer Patients After Surgery According to the UICC Stage Classification and the General Rules for Gastric Cancer Study by the Japanese Research Society for Gastric Cancer. Ann Surg. 1993;218:47-53.

7. Hartgrink H, van de Velde C, Putter H, Bohnenkamp J, Klein Kranenbarg E, Songun I, et al. Extended lymph node dissection for gastric cancer: who may benefit? Final results of the randomized Dutch gastric cancer group trial. J Clin Oncol. 2004;22:2069-77.

8. Macdonald J, Smalley S, Benedetti J, Hundahl S, Estes $\mathrm{N}$, Stemmermann G, et al Chemoradiotherapy after surgery compared with surgery alone for adenocarcinoma of the stomach or gastroesophageal junction. N Engl J Med. 2001;345:725-9. 
REGISTRO DE EVALUACIÓN DE TRATAMIENTO DE CÁNCER GÁSTRICO EN CHILE (REGATE): CARACTERÍSTICAS...

9. Sakuramoto S, Sasako M, Yamaguchi T, Kinoshita T, Fujii M, Nashimoto A, et al. Adjuvant Chemotherapy for Gastric Cancer with S-1, an Oral Fluoropyrimidine. N Engl J Med. 2007;357:1810-20.

10. Cunningham D, Allum W, Stenning S, Thompson J, Van de Velde C, Nicolson M, et al. Perioperative Chemotherapy versus Surgery Alone for Resectable Gastroesophageal Cancer, N Engl J Med. 2006;355:11-20.

11. NCCN Practice Guidelines in Oncology Gastric Cancer v.2.2009, The NCCN Clinical Practice Guidelines in Oncology ${ }^{\mathrm{TM}}$ (C) 2009 National Comprehensive Cancer Network, Inc. Disponible en NCCN.org.

12. Wagner A, Grothe W, Haerting J, Kleber G, Grothey A, Fleig W. Chemotherapy in advanced gastric cancer: a systematic review and meta-analysis based on aggregate data. J Clin Oncol. 2006;24:2904-9.
13. Ter-Ovasenov M, Bang Y, Yalcin S, Roth A, Zalcberg J, Soloviev V, et al. Registry of gastric cancer treatment evaluation (REGATE): Baseline characteristics of 10.299 patients from 22 countries. J Clin Oncol. 2009;27:15s, (suppl; abstr 4575).

14. Heise K, Bertran E, Andia M, Ferreccio C. Incidence and survival of stomach cancer in a high-risk population of Chile. World J Gastroenterol. 2009;15:1854-62.

15. Csendes A, Burdiles P, Braghetto I. Resecabilidad y mortalidad operatoria de la gastrectomía subtotal y total en pacientes con cáncer gástrico avanzado, entre 1969 y 2004. Rev Med Chile 2006;134:426-32.

16. García C, Benavides C, Apablaza S, Rubilar P, Covacevich $\mathrm{S}$, Peñaloza $\mathrm{P}$, et al. Resultados del tratamiento quirúrgico del cáncer gástrico: Análisis de 423 casos. Rev Med Chile 2007:135:687-95. 